\title{
Test Battery Approach to Assess an Individual with Cerebral Venous Sinus Thrombosis: A Speech Language Pathologist's Perspective
}

\author{
Sneha Mareen Varghese Akhila P. A \\ Department of Speech Language studies, Dr. S. R. Chandrasekhar Institute of Speech and \\ Hearing, Bengaluru, India
}

\section{Keywords}

Cerebral venous sinus thrombosis - Speech language pathologist · Assessment .

Cognitive-linguistic profile

\begin{abstract}
Cerebral venous sinus thrombosis (CVST) is an uncommon stroke that has a varied clinical profile. Quite often, speech language pathologists limit the assessment of these patients to a language assessment alone. Subsequently, it is possible that they may miss out certain relevant information which can aid them in their intervention. The aim of the current study was to highlight the relevance of adopting a test battery approach to assess a patient diagnosed with CVST. Here, we present a test battery approach to assess a 43-year-old patient who reported to us after CVST. The test battery included various formal and informal measures. Apart from the routine measures, an additional test for assessing the cognitive linguistic profile was included in the battery which yielded us significant information regarding the patient. Thus, we concluded that speech language pathologists should follow a test battery approach to get a clear idea about the clinical profile of the patient, to avoid misdiagnosis, and also to provide suitable intervention.
\end{abstract}

\section{Introduction}

Cerebral venous sinus thrombosis (CVST) is an uncommon stroke which results when there is an occlusion in the cerebral venous drainage system. There are 2 systems of venous drainage: superficial and deep [1]. CVST can result if thrombosis occurs in any of 
these drainage systems. Normally, blood from the brain drains through smaller to larger cerebral veins. These larger veins empty into dural sinuses which themselves are drained by the internal jugular veins. However, in certain cases, this process is disturbed leading to CVST.

Pathogenesis of sinus thrombosis can be explained using 2 different mechanisms: thrombosis of the cerebral veins and thrombosis of the major veins. The first mechanism, thrombosis of cerebral veins, leads to localized edema of the brain and venous infarction. Edema can be due to an ischemia or due to a disruption in the blood-brain barrier causing a leakage of blood plasma into the interstitial space. The second mechanism causes an increase in intracranial pressure as a result of occlusion of the major veins. These 2 mechanisms lead to a wide spectra of symptoms which makes the diagnosis difficult [2]. Most often its diagnosis is delayed due to lack of apparent symptoms. The common symptom of CVST is headache, and it usually co-occurs with many other neurological signs and symptoms. It has also been reported that the clinical presentation of stroke from venous thrombosis is different from arterial thrombosis. It may present with acute, subacute, or chronic clinical presentation. Recently, one of the studies reported that among the 48 patients with CVST, onset was acute in $44 \%$, subacute in $35 \%$, and chronic in $21 \%$ [3].

The clinical presentation of CVST varies in accordance with mode of onset, time interval between onset and clinical presentation, and primary condition that led to this disease. The signs and symptoms of CVST extend over hours to several weeks. A recent Dutch-European study reported that these patients exhibited severe headache $(95 \%)$, focal seizures with or without generalization (47\%), unilateral or bilateral paresis $(43 \%)$, and papilledema (41\%). Focal neurological deficit may result depending on the area involved [4]. In some cases of CVST, cranial nerve syndromes are also indicated. These patients may present vestibular pathology, unilateral deafness, double vision, and facial paresis. Additionally, aphasia, dizziness, and chorea are also reported [5].

The literature reports of speech disturbances, language disturbances, and readingwriting deficits in CVST patients. However, clinical manifestation of these problems varied with the area of insult. Speech disturbances were indicated in superior sinus thrombosis with a small venous infarct [6], intracranial hypertension in occlusions of lateral sinus [7], aphasia in occlusions of the left transverse sinus [8], posterior alexia in sigmoid sinus thrombosis [9], and acute micrographia and hypophonia in case of deep venous sinus thrombosis [10]. Other characteristics observed were slurred speech, hoarseness, nasal twang, swallowing difficulties, restricted functions of articulators, tinnitus, unilateral hearing problems, and depressed levels of consciousness [11].

It is clear from the review that assessment of post-morbid skills of patients diagnosed with CVST is challenging. It is primarily due to the variations in the clinical profile. A recent study reported the speech and language profile of 7 patients with CVST [12]. Interestingly, all 7 patients had a varied clinical profile. In that study, 5 out of 7 patients presented with noticeable impairments in either speech or language and swallowing. These impairments were due to dysarthria, aphasia, and dysphagia which were associated with CVST. Notably, one of the patients exhibited cognitive impairment on MMSE. However, no further testing was carried out to understand the impact of CVST on his cognitive linguistic skills. Considering the wide spectra of characteristics as evidenced in the literature, a test battery approach in assessment was followed in the current study. It is important that the speech language pathologist (SLP) adopts a test battery approach to avoid underdiagnosis of these patients. Hence, the present study was aimed at highlighting the need for adopting a test battery for assessment of a patient with CVST by an SLP.

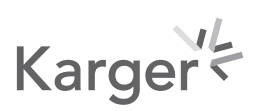




\section{Case Report/Case Presentation}

Ms. X, a right handed, 43-year-old bilingual reported with speaking and writing difficulties. She was a practicing gynecologist. Reportedly, she experienced severe headache followed by repeated convulsions. Medical attention was sought immediately. She was on medications for 6 months. Six months later, she presented memory disturbances for which she consulted a neuropsychiatrist. She reported of upper limb weakness which lasted for 2 weeks. MRI with magnetic resonance angiogram of the brain revealed multiple hyperintense foci in both frontal lobes and mild irregularity of the left transverse sinus with signal void and was advised medications. As reported, she ceased the medications by herself which resulted in speaking difficulties. A more recent MRI of the brain revealed chronic cerebral venous thrombosis with left occipital lobe subacute to chronic infarcts after cerebral sinus thrombosis. Audiological evaluation revealed normal hearing sensitivity in both ears. But, she complained of tinnitus.

\section{Material and Procedures}

Ms. X was subjected to assessment sessions which extended for $3 \mathrm{~h}$ on 4 consecutive days. To ensure that Ms. $\mathrm{X}$ is alert and active, she was given adequate breaks whenever she felt tired or inactive. Assessment was conducted by SLPs who have experience working with adults with neurogenic communication disorders. Assessment protocol was planned based on the areas reported to be affected in individuals with CVST. A case history of Ms. X was collected to gather information on her medical and family history. After collecting history of her illness, Ms. X was subjected to a battery of tests including the Western Aphasia Battery [13] to assess language competence, Manipal Manual for Cognitive Linguistic Abilities [14] to assess perception, memory, and executive functions in individuals with cognitive linguistic disorders, and Mini-Mental State Examination [15] to screen for cognitive impairment. In addition, oral peripheral mechanism examination (OPME) and verbal fluency assessment were carried out. An oral peripheral mechanism examination assesses the structural adequacy and functional integrity of articulators during speech and nonspeech activities. Verbal fluency assesses the information retrieval from memory. Successful retrieval requires executive control over various cognitive processes such as attention, inhibition, and working memory. Semantic and phonemic fluency was assessed using category verbal fluency (fruits and vegetables) and F-A-S [16], respectively. For each task, 60-s duration was given. The Clock Drawing Test [17] was also performed to assess visuospatial and constructional abilities of Ms. X. All the aforementioned assessment was carried out after obtaining a written consent from Ms. X to disseminate the findings.

\section{Results}

\section{Case History}

Ms. X served as the informant for the case history. Case history revealed positive family history for Kawasaki disease and autoimmune disorders. She communicated verbally in 2-3 word phrases. No motor difficulties were reported and observed. She has not been working for the past 6 months for her deficits in communication did not allow her to return to work. Her rate of speech was slow, and she used linguistic mazes extensively. She exhibited significant word-finding difficulties and occasional neologisms. Notably, she could provide sufficient information with cues from the diagnostician.

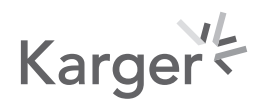




\section{Oral Peripheral Mechanism Examination}

A detailed examination of the oral cavity including size, symmetry, range, speed, accuracy, and coordination of the articulators like lip, jaw, tongue, palate, and velum was performed. All the articulators were observed to be normal with respect to its structure and function.

\section{Verbal Fluency}

Ms. X produced 3 fruits and vegetables with many perseverations in the semantic fluency tasks. In the phonemic fluency tasks, she could name 4 words with "f" as the initial sound (forgot, forward, foot, and fair), 3 words with "a" as the initial phoneme (apple, anyway, and another), and 3 words starting with "s" as the initial phoneme (safe, see, and sarah) which showed a defective word fluency performance indicating lexical retrieval deficits.

\section{Clock Drawing Test}

Ms. X followed the directions and executed the tasks correctly which indicated good visual memory and abstract ability.

\section{Western Aphasia Battery}

Ms. X presented with word-finding difficulties and a longer reaction time after the presentation of a stimulus. Auditory verbal comprehension was nearly preserved except that she could not follow complex sequential commands. Object naming was comparatively better than word fluency and sentence completion tasks. She could repeat sentences up to 3 -word level without any struggle. Her handwriting was legible. Reading aloud and comprehension of sentences were preserved. She scored less on spelled word recognition and spelling tasks. She exhibited spelling errors, micrographia, poor grammatical structure, and lack of cohesion in the written narration task. Spelling errors were noted for irregular words and regular words on dictation. In addition, she could not write all the alphabets in a sequence and could not write 2-digit numbers on dictation. She could perform relatively better in all the tasks under apraxia and constructional, visuospatial, and calculation section.

Based on the Western Aphasia Battery scores (Table 1), Ms. X has nonfluent aphasia. Taxonomical classification revealed that Ms. X exhibited a conduction aphasia. Aphasia quotient was 54.4 which indicated moderate severity.

\section{Mini-Mental State Examination}

Ms. X indicated difficulty while performing backward counting/reverse spelling and repetition of sentences. A total score of 22 was obtained, which was suggestive of a mild level of cognitive impairment.

\section{Manipal Manual for Cognitive Linguistic Abilities}

The raw scores obtained for each section of MMCLA are represented in Table 2. The summary of observations of each section is discussed below.

\section{Perception}

Ms. X indicated poorer scores on auditory letter and word search as the level of complexity increased. Specifically, she found it difficult to identify letters and clusters as the length of the letters and words in the list increased. Poor performance was noted for auditory-related word search and word search from a passage. In addition, she exhibited difficulty in naming an object with the cues provided auditorily. She was able to perform this task only after

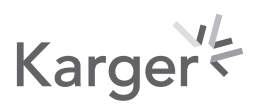


Table 1. Raw scores obtained for various subsections of the Western Aphasia Battery

\begin{tabular}{|c|c|c|}
\hline Subsections & Patient's score & Maximum score \\
\hline \multicolumn{3}{|l|}{ Spontaneous speech } \\
\hline Information content & 5 & 10 \\
\hline Fluency & 5 & 10 \\
\hline \multicolumn{3}{|l|}{ Comprehension } \\
\hline Yes/no questions & 57 & 60 \\
\hline Auditory word recognition & 57 & 60 \\
\hline \multirow[t]{2}{*}{ Sequential command } & 36 & 80 \\
\hline & 150 & 200 \\
\hline Repetition & 48 & 100 \\
\hline \multicolumn{3}{|l|}{ Naming } \\
\hline Object naming & 33 & 60 \\
\hline Word fluency & 2 & 20 \\
\hline Sentence completion & 6 & 10 \\
\hline \multirow[t]{2}{*}{ Responsive speech } & 8 & 10 \\
\hline & 69 & 100 \\
\hline \multicolumn{3}{|l|}{ Reading and writing } \\
\hline Reading & 74 & 100 \\
\hline Writing & 55 & 100 \\
\hline Praxis & 60 & 60 \\
\hline \multicolumn{3}{|l|}{ Construction } \\
\hline Drawing & 24 & 30 \\
\hline Block design & 9 & 9 \\
\hline Calculation & 24 & 24 \\
\hline
\end{tabular}

providing all the cues related to the object. In the visual perception section, she scored poorer of visual feature search where she had to identify items based on a feature. Her performance on visual abstract identification was comparatively better.

\section{Short-Term Memory}

Ms. X was able to perform the auditory word scan and visual letter scan tasks without any difficulty. However, a poor performance was observed for both visual and auditory memory span tasks when the task was to recollect the words presented along with a set of 3 or more sentences.

\section{Working Memory}

Performance on auditory word retrieval, letter, and number recall was adequate for a set of 3 words, letters, or numbers. But, there was a decrease in performance with an increase in number of stimulus items in a set. Similar trend was noted for all tasks of auditory and visual processing skills.

Decision, Judgment, and Reasoning

Making conclusion from a pair of sentences was difficult when presented both auditorily and visually. Reasoning skills were adequate for both auditory and visual conditional reasoning.

\section{Problem Solving}

Problem solving skills were adequate. Organization of tasks in the order of execution was difficult with sentence complexity. Domains assessing perception and execution task show a mild level of impairment, and memory predominantly working memory shows a moderate level of impairment which further indicates cognitive linguistic deficit. 
Table 2. Raw scores obtained for various sections of Manipal Manual of Cognitive Linguistic Abilities

\begin{tabular}{|c|c|c|c|}
\hline Domains & Subsections & Patient score & Maximum score \\
\hline \multirow[t]{11}{*}{ Perception } & I. A. Auditory letter and word search & 24 & 41 \\
\hline & I. A7. Auditory-related word search & 1 & 5 \\
\hline & I. A8. Auditory word search & 1 & 4 \\
\hline & I. B. Visual letter search & 20 & 20 \\
\hline & Visual picture search & 5 & 5 \\
\hline & Visual number search & 15 & 15 \\
\hline & Visual word search & 5 & 5 \\
\hline & I. C. Auditory object search & 17 & 20 \\
\hline & I. D. Visual action search & 4 & 5 \\
\hline & I. E. Visual feature search & 1 & 5 \\
\hline & I. F. Abstract identification & 22 & 25 \\
\hline \multirow[t]{4}{*}{ Short-term memory } & II. A. Auditory word scan & 5 & 5 \\
\hline & II. B. Visual letter scan & 10 & 10 \\
\hline & II. C. Auditory memory span & 6 & 14 \\
\hline & II. D. Visual memory span & 8 & 14 \\
\hline \multirow[t]{10}{*}{ Working memory } & III. A. Auditory retrieval & & \\
\hline & Auditory word retrieval & 11 & 20 \\
\hline & Auditory letter and number recall & 1 & 5 \\
\hline & Auditory word list recall & 3 & 15 \\
\hline & Auditory delayed sentence recall & 12 & 15 \\
\hline & III. B. Visual retrieval & & \\
\hline & Visual picture recall & 1 & 5 \\
\hline & Visual letter and number recall & 0 & 5 \\
\hline & Visual word list recall & 1 & 15 \\
\hline & Visual delayed sentence recall & 12 & 15 \\
\hline \multirow{4}{*}{$\begin{array}{l}\text { Decision, judgment, } \\
\text { and reasoning }\end{array}$} & IV. A. Syllogism (auditory) & 5 & 10 \\
\hline & IV. B. Syllogism (visual) & 2 & 5 \\
\hline & IV. C. Auditory conditional reasoning & 5 & 5 \\
\hline & IV. D. Visual conditional reasoning & 4 & 5 \\
\hline \multirow[t]{7}{*}{ Problem solving } & V. A. Visual divergent production & 3 & 5 \\
\hline & V. B. Auditory divergent production & 11 & 20 \\
\hline & V. C. Auditory mean-end analysis & 4 & 5 \\
\hline & V. D. Visual mean-end analysis & 5 & 5 \\
\hline & V. E. Auditory analogy & 4 & 5 \\
\hline & V. F. Visual analogy & 5 & 5 \\
\hline & V. G. Sequential task analysis & 1 & 10 \\
\hline
\end{tabular}

\section{Conclusions}

The current study followed a test battery approach to assess a patient who reported after CVST. Based on the literature, it was evident that the clinical profile of a patient with CVST varies extensively [18], and hence a test battery for assessment was opted. Case history of the patient provided significant information on the family history of medical ailments. In addition, it helped the researchers in understanding the medical history of the patient. Case history 
also provided information on tinnitus, which is a frequent complaint after CVST [11]. On oral peripheral mechanism examination, it was clear that Ms. X had normal structure and function of articulators. The possibility of dysarthria was thus ruled out. The previous research also reported of normal structure and function in individuals with mild cognitive impairment after CVST [12]. The results of verbal fluency revealed that the lexical semantic organization of the patient is affected.

Interestingly, WAB yielded a diagnosis of conduction aphasia. However, it was evident from the scores that the patient had significant difficulties in repetition due to her inability to retain sentences in memory. Thus, poorer scores on repetition could be attributed to working memory deficits associated with individuals with CVST. It is possible that these patients have lesser scores on naming due to their lexical retrieval deficits. WAB gave the researchers an insight into the patient's reading and writing deficits as well. Micrographia was noticed [10]. Since the researchers felt that the difficulty in repetitions was mainly due to deficits in memory, a detailed cognitive linguistic assessment was carried out. Further, MMSE of the patient yielded a score denoting mild cognitive impairment. Notably, deficits were observed primarily in memory-related tasks. This was evident irrespective of the mode of stimulus presentation. Accordingly, she indicated difficulties in auditory perception tasks which placed demands on working memory. Interestingly, auditory-based tasks yielded poorer scores compared to the visual-based tasks. This needs to be explored further by evaluating her auditory processing skills.

Thus to conclude, the current study alarms the speech language pathologists to look beyond the speech and language skills of patients reported with a loss of language after CVST. Accordingly, this study stresses on including assessment of cognitive-linguistic skills of patients reported with a history of CVST, not the routine assessment tools or procedures alone. This will definitely aid the clinicians in tailor-making the intervention protocol for the patients with CVST. Future studies with larger sample size are recommended.

\section{Statement of Ethics}

The authors have no ethical conflicts to disclose. The authors confirm obtaining written informed consent from the subject for publication of the manuscript including case history and test findings.

\section{Conflict of Interest Statement}

The authors have no conflicts of interest to declare.

\section{Funding Sources}

The authors have no funding source to disclose.

\section{Author Contributions}

The assessment protocol was planned by the first author. The clinical evaluation was carried out by both the authors. The manuscript was drafted by the first author with minimal support from the second author.

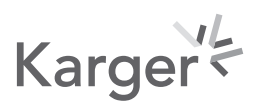




\section{References}

1 Alvis-Miranda HR, Milena Castellar-Leones S, Alcala-Cerra G, Rafael Moscote-Salazar L. Cerebral sinus venous thrombosis. J Neurosci Rural Pract. 2013;4(4):427-38.

2 Bousser MG, Barnett HJM. Cerebral venous thrombosis. 4th ed. In: Mohr JP, Choi DW, Grotta JC, Weir B, Wolf PA, editors. Stroke-pathophysiology diagnosis and management. Philadelphia (USA): Churchill Livingstone; 2004. p. 301-25.

3 Terazzi E, Mittino D, Rudà R, Cerrato P, Monaco F, Sciolla R, et al. Cerebral venous thrombosis: a retrospective multicentre study of 48 patients. Neurol Sci. 2005;25(6):311-5.

4 de Bruijn SF, de Haan RJ, Stam J. Clinical features and prognostic factors of cerebral venous sinus thrombosis in a prospective series of 59 patients. For The Cerebral Venous Sinus Thrombosis Study Group. J Neurol Neurosurg Psychiatry. 2001;70(1):105-8.

5 McElveen WA. eMedicine world medical library. Cerebral venous thrombosis. 2005.

6 Gupta RK, Jamjoom AA, Devkota UP. Superior sagittal sinus thrombosis presenting as a continuous headache: a case report and review of the literature. Cases J. 2009 Dec 21;2(12):9361. Available from: https://casesjournal.biomedcentral.com/.

7 Sood N, Sood N, Talkad A. A case of worsening deep cerebral venous sinus thrombosis managed by intrasinus thrombolysis. Case Rep Neurol Med. 2011 Oct 12;2011:272841.

8 Leys D, Cordonnier C. Cerebral venous thrombosis. Update on clinical manifestations, diagnosis and management. Ann Indian Acad Neurol [Internet]. 2008;11(5);79-87. Available from: http://wwww.annalssofian.org.

9 Thomas P, Keightley A, Kamble R, Gunasekera N, Johnson MR. Sigmoid sinus thrombosis presenting with posterior alexia in a patient with Behçet's disease and polycythaemia: a case report. J Med Case Rep. 2008 May 23;2:175.

10 Murray BJ, Llinas R, Caplan LR, Scammell T, Pascual-Leone A. Cerebral deep venous thrombosis presenting as acute micrographia and hypophonia. Neurology. 2000 Feb 8;54(3):751-3.

11 Byju N, Jose J, Saifudheen K, Gafoor VA, Jithendranath P. Cerebral venous thrombosis presenting as multiple lower cranial nerve palsies. Indian J Crit Care Med. 2012 Oct-Dec;16(4):213-5.

12 Vandana VP. Speech-language profile, neurological findings and recovery patterns associated with cerebral sinus thrombosis. Lang India [Internet]. 2014 [cited 2014 Dec 12];14:679-86. Available from: http://www. languageinindia.com.

13 Kertesz A. Western aphasia battery. New York: Grune and Stratton; 1982.

14 Mathew MM, Bhat JS, Sreya NM, Arora A. Manipal manual of cognitive linguistic ability. Karnataka: Manipal university press; 2013.

15 Folstein MF, Folstein SE, McHugh P. "Mini Mental state": a practical method for grading the cognitive state of patients for the clinician. J Psychiatr Res. 1975;12:189-98.

16 Agrell B, Dehlin O. The clock-drawing test. Age Ageing. 1998;27(3):399-403.

17 Spreen 0, Benton AL. Neurosensory center comprehensive examination for aphasia. Victoria (BC): University of Victoria, Neuropsychology Laboratory; 1977.

18 Mehndiratta MM, Garg S, Gurnani M. Cerebral venous thrombosis--clinical presentations. J Pak Med Assoc. 2006 Nov;56(11):513-6. 